\title{
ENSAYO DE PROCEDENCIAS Y PROGENIES NORTEAMERICANAS DE PINO OREGON Pseudotsuga menziesii (Mirb.) Franco
}

\author{
Iván Quiroz M. ${ }^{1}$, Marco Barrientos A. ${ }^{1}$ y Marta González O. ${ }^{1}$
}

\section{RESUMEN}

En el año 2000 el Instituto Forestal chileno (INFOR) estableció ensayos de procedencias y progenies de Pseudotsuga menziesii (Mirb.) Franco, pino oregón, en las Regiones de La Araucanía, Los Ríos y Los Lagos, con el objeto de seleccionar aquellas más adecuadas para la forestación y sentar las bases para un programa de mejoramiento genético para esta especie en el país.

Los ensayos contienen una completa colección de procedencias y familias, seleccionadas desde individuos superiores en las áreas de origen de la especie en Norteamérica por la Universidad del estado de Oregón en los Estados Unidos, que incluye 19 procedencias y 671 familias.

Estos ensayos han sido objeto de seguimiento periódico, mediante mediciones de control a las edades de 1, 3, 5 y 10 años, los resultados están siendo evaluados y en este artículo se presenta un avance con los resultados obtenidos para las procedencias en uno de los ensayos, ubicado en el predio Voipir, Región de La Araucanía.

\section{SUMMARY}

Pseudotsuga menziesii provenance-.progeny trials were established by the Chilean Forestry Institute in 2000 in the La Araucanía, Los Ríos and Los Lagos Regions. Main objective is to select appropriate material to use in afforestation plans and to support a genetic improvement program for the species in the country.

A complete provenance -.progeny collection is included, considering 19 provenances and 671 progenies from superior trees through the different natural distribution areas of the species in North America. The collection was provided by the Oregon State University.

The trials have been periodically measured at 1, 3, 5 and 10 years old, the results are under evaluation and this paper provides preliminary results for provenances from one of the trials, located in the Voipir farm, la Araucanía Region. 


\section{INTRODUCCION}

Los programas de diversificación forestal impulsados en el país se han orientado, con importantes resultados, a incorporar nuevas especies al proceso productivo forestal y a profundizar en el conocimiento de aquellas que han mostrado una notable capacidad de crecimiento.

Como consecuencia, se ha logrado identificar algunas especies como prioritarias para contribuir al desarrollo del sector y ser un aporte económico para Chile. Un ejemplo claro de esto es el caso del pino oregón, Pseudotsuga menziesii (Mirb.) Franco, conífera norteamericana considerada como una significativa fuente de madera en la economía mundial y ampliamente difundida en el mundo por la calidad y versatilidad de esta madera.

Esta especie constituye para los productores una opción de diversificación de la oferta de productos al mercado nacional e internacional y, además, representa una alternativa que permite optimizar la productividad de suelos en los cuales las especies tradicionalmente empleadas no se desarrollan satisfactoriamente.

Dado que el nivel de conocimiento y desarrollo tecnológico sobre pino oregón dista considerablemente del alcanzado para pino y eucalipto, INFOR por medio de diversos proyectos ha sentado las bases de un programa de mejoramiento genético de largo plazo, el que ha incluido la selección en Chile de áreas productoras de semillas, la multiplicación clonal de individuos selectos, y el establecimiento de huertos semilleros clonales y de ensayos de procedencias y progenies, entre otras investigaciones sobre la especie.

Como parte de este programa de investigación sobre pino oregón, INFOR estableció en el año 2000 ensayos de procedencia-progenie en el predio Voipir, de Forestal Voipir, Villarrica, Región de La Araucanía, y en el predio Naltahue, de Agrícola y Forestal Naltahue, Lanco, Región de Los Ríos, y ensayos de procedencias en Tehualda, Osorno, Región de Los Lagos, en superficies de 14 ha los dos primeros y de 4 ha el último.

Estos ensayos constituyen una de las más completas colecciones de procedencias y progenies norteamericanas de la especie existentes en Chile, conformada por 19 procedencias y 671 familias y originada en una colección de semillas, obtenidas desde árboles plus, proporcionada por la Oregon State University de los Estados Unidos. La viverización de plantas la efectuó BIOFOREST SA y el establecimiento de los ensayos lo realizó INFOR.

Estos ensayos han sido objeto de seguimiento con mediciones de control al primer año de edad y a los 3, 5 y 10 años, y los resultados de procedencias y progenies están siendo evaluados. En el presente artículo se entrega un avance de resultados para las procedencias en el ensayo del predio Voipir, Región de La Araucanía.

\section{OBJETIVOS}

El objetivo general es la evaluación de la respuesta a diferentes sitios, entre las Regiones de La Araucanía y Los Lagos, de procedencias y progenies de pino oregón, con el fin de seleccionar aquellas más adecuadas para la forestación con esta especie. Se busca además 
sentar las bases para un programa de mejoramiento genético de esta especie en el país.

El objetivo del presente trabajo es adelantar resultados obtenidos con las procedencias en uno de los ensayos establecidos por esta línea de investigación, en el predio Voipir, en la Región de La Araucanía.

\section{ANTECEDENTES DE PINO OREGÓN}

En Chile, pino oregón ha encontrado un ambiente muy propicio para su desarrollo entre las Regiones de la Araucanía y Aysén, totalizando al año 2009 una superficie plantada de 16.676 ha, que lo posiciona como la tercera conífera más plantada en el país, por debajo de pino radiata y pino ponderosa (Pinus ponderosa) (INFOR, 2010).

Pino oregón pertenece a la familia de Pinaceae y es también conocido como Douglas Fir, abeto Douglas, o simplemente Douglas. Es una conífera originaria del occidente de Norteamérica y ocupa un área muy vasta que va desde México $\left(12^{\circ} \mathrm{LN}\right)$ al estado canadiense

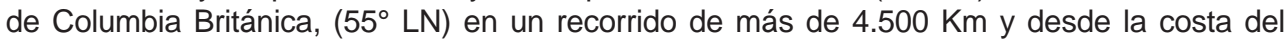
Pacífico hasta las vertientes de las montañas rocosas.

La gran extensión de su área da lugar a la existencia de grandes variaciones genéticas. Botánicamente se distinguen dos variedades: Pseudotsuga menziesii var. menziesii y Pseudotsuga menziesii var. glauca. La primera habita en las regiones costeras del Pacífico, en los Estados de California, Washington y Oregón, en Estados Unidos, y Columbia Británica, en Canadá. Esta variedad es la que se ha introducido en países como Alemania, Francia, España, Nueva Zelandia y Chile (Quiroz y Rojas, 2003). La segunda variedad, de follaje más azulado, ocupa un área netamente más continental, con menores precipitaciones e inviernos más rigurosos, tolerando altitudes muy elevadas. Su menor crecimiento y su sensibilidad a la caída de las acículas (needlecast, Rhabdocline pseudotsugae) hace que no se la emplee cuando el clima es atlántico (Vega et al., 1998).

El pino de oregón crece en una gran variedad de climas, desde oceánicos a continentales, con reparto uniforme de precipitaciones o con sequía estival marcada. Tolera muy bien los fríos invernales y la nieve, pero puede considerarse sensible a heladas tardías. En regiones en que se producen heladas de primavera estas pueden causar daños importantes cuando se usan procedencias que dan lugar a un brote muy temprano. En su área de origen, Pseudotsuga menziesii var. menziesii alcanza altitudes máximas de 1.800 metros en California, 1.500 metros en su área central y 800 metros en la Columbia Británica. Ello indica que la altitud máxima depende de la latitud correspondiente (Vega et al., 1998).

Esta especie es medianamente exigente en cuanto a suelos, prefiriendo terrenos profundos, de textura ligera y fértil. No debe plantarse en suelos muy superficiales o muy pobres. Los suelos con menor profundidad, que presenten una capa compacta superficial o encharcamiento, no son aptos para repoblar con la especie pues limitan excesivamente el crecimiento o producen árboles mal enraizados susceptibles de derribos por viento o nieve, por lo que tolera mal las texturas pesadas. La pedregosidad superficial no constituye una limitación definitiva si el sistema radical puede tener acceso a suficiente cantidad de tierra fina. En general requiere unas buenas condiciones para la aireación de las raíces. La falta de aireación de las 
raíces provoca amarillamiento, lentitud del crecimiento e incluso la muerte de las plantas. Por ello los suelos más convenientes deben tener una textura ligera; franco-arenosa, franco-limosa o arenosa, y deben evitarse los suelos con mucho contenido en arcilla (más de un 15-20\%). Los niveles de $\mathrm{pH}$ del suelo más favorables oscilan entre 4,0 y 6,0. En cuanto a fertilidad, es una especie moderadamente exigente (Vega et al., 1998).

En todas las especies forestales usadas en plantaciones, adquiere gran importancia el uso de una planta proveniente de semilla de origen adecuado, para asegurar que estas se adapten perfectamente al lugar de plantación y presenten una supervivencia y crecimientos óptimos.

El caso de pino oregón no es una excepción, más aún cuando en su lugar de origen posee una amplia distribución, que da lugar a un gran número de procedencias o regiones geográficas, y cada una de estas procedencias tiene una respuesta muy distinta al medio (Vega et al., 1998; Quiroz y Rojas, 2003).

Las procedencias pueden tener una gran relevancia en aspectos tales como, calidad de la madera, resistencia a plagas y enfermedades, crecimiento, otros.

En Nueva Zelanda se han realizado ensayos de procedencias de pino oregón que han determinado que los mejores orígenes son probablemente del sur de Washington y el norte de California (Cown, 1992). Miller y Knowles (1994), señalan que, en dos ensayos de procedencias evaluados a los 6 y 13 años, concluyeron que las mejores procedencias resultaron ser las de la Costa de California y el sur de Oregón.

Ensayos realizados en España, con 91 procedencias de esta especie desde su distribución natural, arrojaron que 44 de ellas presentaron resultados promisorios y correspondían a las del suroeste de British Columbia, oeste de Washington y noroeste de Oregón (Toval et al., 1993).

En cuanto a las procedencias de mejor desempeño en Chile, aún no hay experiencias muy concluyentes. Droppelmann (1986) evaluó un ensayo establecido en el año 1967 en Valdivia donde se probaron 10 procedencias de pino oregón de los Estados de Washington y Oregón y este autor concluye que las mejores procedencias son las ubicadas en la costa de ambos estados.

Aguirre y Wrann (1989) evaluaron tres ensayos de pino oregón establecidos en el año 1968, cuyas procedencias provenían de poblaciones costeras del noroeste de Estados Unidos y de British Columbia en Canadá, concluyendo que las poblaciones costeras principalmente del Estado de Washington fueron las que mejor se adaptaron a la zona centro sur de Chile, tanto para zonas de cordillera como de costa.

\section{MATERIAL Y MÉTODO}

\section{Antecedentes del Lugar de Ensayo}

El ensayo está ubicado a 12 km de Villarrica, Región de La Araucanía. Se encuentra en el Fundo Voipir, perteneciente a la Empresa Forestal Voipir S.A. 
El área corresponde a una posición de valle andino y cordillera baja a una altitud aproximada de $325 \mathrm{msnm}$.

El régimen térmico varía, en promedio, entre una máxima de enero de $21,7^{\circ} \mathrm{C}$ y una mínima de julio de $4,3^{\circ} \mathrm{C}$. El periodo libre de heladas es de 47 días, con un promedio de 31 heladas por año.

El sector registra anualmente 632 días - grado y 3.094 horas frío. El régimen hídrico registra una precipitación media anual de 2.454 a $3.477 \mathrm{~mm}$, con un déficit hídrico de $60 \mathrm{~mm}$ y sin periodo seco (Santibáñez y Uribe, 1993).

El ensayo se encuentra en la Serie de Suelos Correltúe, que pertenece a la Familia de Suelos Lastarria. La temperatura del suelo media anual fluctúa entre $11^{\circ} \mathrm{C}$ y $12^{\circ} \mathrm{C}$, mientras que la media de julio entre $8^{\circ} \mathrm{C}$ y $9^{\circ} \mathrm{C}$ y la media máxima de enero entre $20^{\circ} \mathrm{C}$ y $22^{\circ} \mathrm{C}$.

En cuanto a la caracterización del perfil edáfico de la serie, se considera la presencia de 5 horizontes genéricos, representados por cenizas volcánicas modernas, desarrollados sobre complejos metamórficos (Tosso, 1985).

\section{Procedencias en Ensayo}

En la selección de las procedencias fueron considerados los aspectos de clima y suelo de las Regiones del Maule a Los Lagos.

Las colecciones de semillas fueron realizadas por la Oregón State University, siguiendo las recomendaciones del Dr. Thomas Adams, del Área de Silvicultura de esta universidad norteamericana. La colecta de semillas se efectuó desde árboles plus de la especie en sus diferentes áreas de origen.

La colección de procedencia-progenie fue enviada por la Oregon State University a BIOFOREST SA en Chile, empresa que efectuó la viverización de plantas, y posteriormente INFOR estableció los ensayos en el año 2000, efectuando igualmente el seguimiento de estos.

En el Cuadro $N^{\circ} 1$ se entrega el detalle de procedencias y familias incluidas en el ensayo ubicado en el predio Voipir, Región de La Araucanía. 


\section{Cuadro $N^{\circ} 1$ \\ PROCEDENCIAS Y FAMILIAS DE PINO OREGÓN EN ENSAYO PREDIO VOIPIR AÑO 2000}

\begin{tabular}{|c|l|l|c|l|l|}
\hline $\begin{array}{c}\text { Región o } \\
\text { Grupo }\end{array}$ & Procedencia & $\begin{array}{l}\text { Zona Semilla } \\
\text { EE.UU }\end{array}$ & $\begin{array}{c}\text { Simbología } \\
\text { Procedencia }\end{array}$ & $\begin{array}{l}\text { Familias } \\
\left(\mathbf{N}^{\circ}\right)^{2}\end{array}$ & $\begin{array}{l}\text { Elevación } \\
\text { (msnm) }\end{array}$ \\
\hline \multirow{4}{*}{ Costa Sur } & Chetco & $090-082$ & A4 & $1-40$ & $<500$ \\
& Gold Beach / Powders & $081-072$ & A5 & $41-80$ & $<500$ \\
& Mapleton & 062 & A6 & $81-120$ & $<300$ \\
& Walport & 061 & A7 & $121-151$ & $<300$ \\
\hline \multirow{5}{*}{ Costa Norte } & Umpqua coast & 071 & B4 & $152-191$ & $150-500$ \\
& Noti & $062-252-262 S$ & B5 & $192-231$ & $150-550$ \\
& Burnt Woods & $061-252-262 N$ & B6 & $232-270$ & $150-600$ \\
& Vernonia & 052 & B7 & $271-310$ & $<600$ \\
& Shelton & $231-222-241-030$ & B8 & $311-342$ & $<150$ \\
\hline \multirow{5}{*}{ Altura } & Oakridge & 482 & C1 & $343-382$ & $300-600$ \\
& Mc Kenzie A & 472 & C2 & $383-417$ & $300-600$ \\
& Sweet Home & 462 & C3 & $418-441$ & $300-600$ \\
& Mt Hood A & $451-452$ & C4 & $442-481$ & $<600$ \\
& Cowlitz A & $430-422$ & C5 & $482-518$ & $<600$ \\
& Snoqualmie & 403 & C6 & $519-558$ & $300-600$ \\
& Skagit & $401-402$ & C7 & $559-598$ & $300-600$ \\
\hline \multirow{2}{*}{ Baja } & Mc Kenzie B & 472 & D1 & $599-638$ & $900-1200$ \\
precipitación & Mt Hood B & $451-452$ & D2 & $639-678$ & $>600$ \\
& Cowlitz B & $430-422$ & D3 & $679-721$ & $750-1050$ \\
\hline Local & Voipir & & & & \\
\hline Total & & & & 672 & \\
\hline
\end{tabular}

(Quiroz y Rojas, 2003)

\section{Diseño Experimental}

El ensayo se estableció con un diseño experimental de bloques al azar.

Como se indica en el Cuadro $N^{\circ} 1$, las procedencias fueron divididas en 4 grupos; zonas de costa sur, costa norte, altura y baja precipitación.

El set costa está formado por 9 procedencias y 314 familias, y el set Interior por 10 procedencias y 358 familias. Se incorporó además una procedencia local, obtenida de la zona de Voipir. 
El ensayo tiene 12 bloques, cada set es de 6 bloques, cada bloque representa una repetición, el cual contiene las parcelas que representan a las familias con un tamaño de 5 plantas cada parcela (Figura $\left.\mathrm{N}^{\circ} 1\right)$.

El modelo planteado considera efectos fijos, bloque y procedencia, y aleatorio, familia dentro de procedencia. Los resultados del análisis de varianza consideran el modelo Tipo III (modelo mixto), que contempla las siguientes fuentes de variación:

- Bloque (efecto fijo ${ }^{3}$ )

- Procedencia (efecto fijo)

- Bloque x Procedencia (efecto fijo)

- Familias dentro de Procedencia (efecto aleatorio) ${ }^{4}$

- Bloque x Familias dentro de Procedencia (efecto aleatorio) (parcela)

El modelo es:

$$
y_{i j k l}=\mu+b_{i}+p_{j}+b(p)_{i j}+f(p)_{j k}+b^{*} f(p)_{i j k}+e_{i j k l}
$$

Donde:

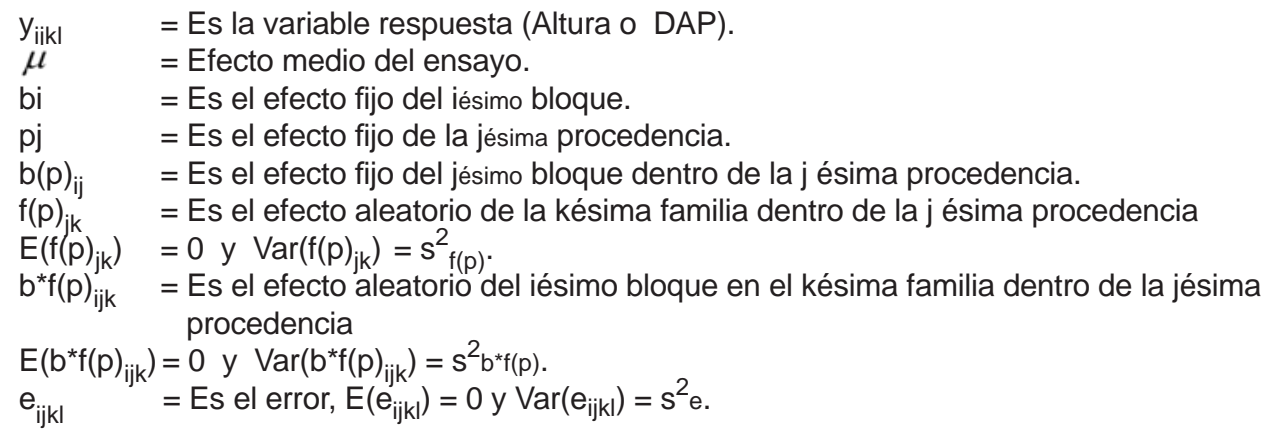

3 Efecto fijo: Cuando se está interesado únicamente en los tratamientos presentes en el ensayo (bloques y procedencias).

4 Efecto aleatorio: Cuando se quiere hacer inferencias respecto a la población de tratamientos, de los cuales solamente una muestra al azar (los tratamientos) están presente en el ensayo (familias). 


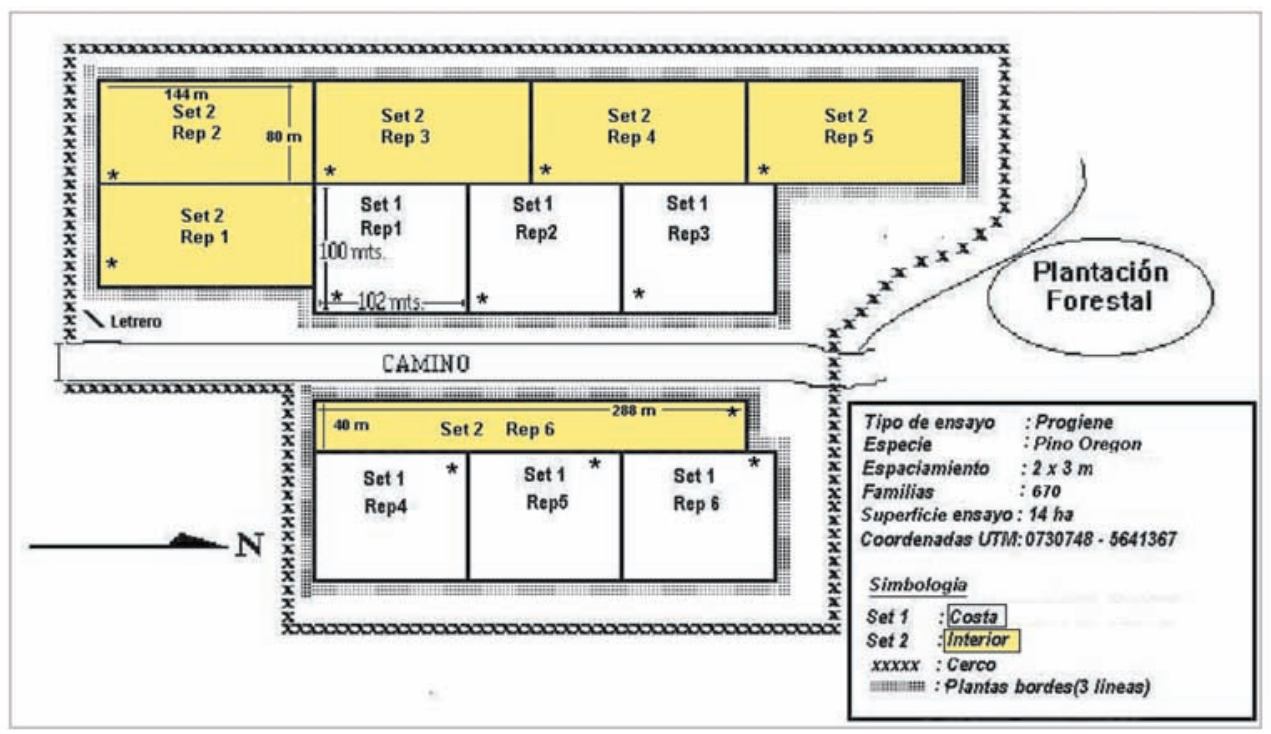

\section{Figura $N^{\circ} 1$ \\ CROQUIS DE DISTRIBUCIÓN ENSAYO PROCEDENCIA - PROGENIE PREDIO VOIPIR 2000}

\section{Establecimiento del Ensayo}

La plantación fue establecida en agosto del año 2000. La densidad de plantación fue de $1.667 \mathrm{pl} / \mathrm{ha}$, a un espaciamiento de $3 \times 2 \mathrm{~m}$, utilizando una superficie total aproximada de 14 hectáreas.

En el establecimiento del ensayo se consideraron las siguientes prácticas culturales:

Control químico de malezas preplantación: Se realizó en la superficie del ensayo un control químico, los productos y dosis aplicadas fueron los siguientes: Roundup líquido 3 L/ha y Atrazina: $3 \mathrm{~kg} / \mathrm{ha}$

Cultivo: Cultivo manual (pala plantadora) por cada hoyo de plantación, de 40 x $40 \mathrm{~cm}$ y una profundidad de $30 \mathrm{~cm}$.

Control de lagomorfos: Previo a la plantación se aplicó insecticida en pastillas para combatir el ataque de liebres y conejos, el producto aplicado fue Phostoxin, aplicando el producto en cada una de las cuevas que estuvieran al interior del ensayo.

Esta aplicación debió repetirse durante la plantación, debido al ataque observado en las 
plantas recién establecidas. A fines del mes de octubre del año 2000, se observó nuevamente un fuerte ataque de liebres y conejos sobre las plantas, para lo cual fue necesario colocar un cerco de malla de $80 \mathrm{~cm}$ de altura desde el nivel del suelo y $40 \mathrm{~cm}$ en profundidad, alrededor de todo el ensayo.

Control químico de malezas post plantación 1: En noviembre del año 2000 se realizó la segunda aplicación química para combatir la aparición de maleza. El producto aplicado fue Velpar: 2,5 kg/ha. Este producto se aplicó considerando una taza de $1 \mathrm{~m}$ de diámetro alrededor de la planta.

Desbrote manual: En diciembre del año 2000 y parte de enero del año 2001 se realizó un desbrote manual con desbrozadora sobre la hilera de plantación para controlar la maleza.

Control químico de malezas post plantación 2: En el año 2001, durante el mes de octubre se realizó un segundo control químico post plantación para controlar la maleza.

Este control fue aplicado entre y sobre hilera, de acuerdo a las siguientes dosis empleando Velpar 0,6 - 0,8 kg/ha y Atrazina: 3,2 L/ha.

Posteriormente las mediciones de seguimiento fueron realizadas entre los meses de abril y mayo de los años 2001, 2003, 2005 y 2010. Las variables evaluadas fueron altura (m) y DAP (cm).

La supervivencia no fue evaluada como variable resúesta, dado que en general en estos ensayos de pino oregón esta ha sido muy alta, por sobre el $80 \%$ al año 10 . Inicialmente se registraron daños provocados por conejos y liebres, pero las plantas fueron respuestas.

\section{RESULTADOS Y DISCUSION}

De acuerdo con los crecimientos observados, se aprecia que el grupo Costa Sur presentó los mayores valores para todos los parámetros evaluados, seguido por Costa Norte y por la procedencia local.

El grupo que presentó los valores más bajos para la altura y DAP fue el de Baja Precipitación, siendo estas diferencias estadísticamente significativas respecto a lo obtenido con los otros grupos (Cuadro $\mathrm{N}^{\circ} 2 \mathrm{y}$ Figura $\mathrm{N}^{\circ} 2$ ). Cabe señalar que las diferencias de altura en el año 2010 entre los grupos de mayor y menor crecimiento fue de 2,36 m (8,12 - 5,76 m) y las diferencias de DAP de 4,31 cm $(12,92-8,61 \mathrm{~cm})$.

Respecto del comportamiento de la procedencia local, esta presentó una altura menor en 1,36 m respecto al Grupo más alto (Costa Sur), en el DAP en tanto esta diferencia fue de $1,73 \mathrm{~cm}$ respecto al Grupo que presento el mayor valor para esta variable, Costa Sur. 


\section{Cuadro $\mathrm{N}^{\circ} 2$}

\section{COMPARACIONES MÚLTIPLES PARA LAS VARIABLES ALTURA Y DAP POR GRUPO}

\begin{tabular}{|c|c|c|c|c|c|c|c|c|c|c|}
\hline GRUPO & $\begin{array}{c}\text { Altura } \\
\mathbf{2 0 0 1} \\
\mathbf{( m )}\end{array}$ & DE & $\begin{array}{c}\text { Altura } \\
\mathbf{2 0 0 3} \\
\mathbf{( m )}\end{array}$ & DE & $\begin{array}{c}\text { Altura } \\
\mathbf{2 0 0 5} \\
\mathbf{( m )}\end{array}$ & DE & $\begin{array}{c}\text { Altura } \\
\mathbf{2 0 1 0} \\
\mathbf{( m )}\end{array}$ & DE & $\begin{array}{c}\text { DAP } \\
\mathbf{2 0 1 0} \\
\mathbf{( c m})\end{array}$ & DE \\
\hline Altura & $0,31 \mathrm{~d}$ & 0,11 & $0,81 \mathrm{~d}$ & 0,37 & $1,92 \mathrm{~d}$ & 0,66 & $6,43 \mathrm{c}$ & 1,77 & $9,94 \mathrm{c}$ & 3,53 \\
\hline Baja Precipitación & $0,23 \mathrm{e}$ & 0,09 & $0,60 \mathrm{e}$ & 0,30 & $1,48 \mathrm{e}$ & 0,61 & $5,76 \mathrm{~d}$ & 1,82 & $8,61 \mathrm{~d}$ & 3,63 \\
\hline Procedencia Local & $0,45 \mathrm{~b}$ & 0,15 & $1,19 \mathrm{c}$ & 0,49 & $2,63 \mathrm{c}$ & 0,84 & $6,76 \mathrm{c}$ & 1,60 & $11,19 \mathrm{~b}$ & 5,58 \\
\hline Costa Norte & $0,40 \mathrm{c}$ & 0,12 & $1,19 \mathrm{c}$ & 0,40 & $2,65 \mathrm{c}$ & 0,68 & $7,92 \mathrm{~b}$ & 1,62 & $12,31 \mathrm{~b}$ & 2,90 \\
\hline Costa Sur & $0,47 \mathrm{~b}$ & 0,14 & $1,34 \mathrm{~b}$ & 0,42 & $2,91 \mathrm{~b}$ & 0,71 & $8,12 \mathrm{a}$ & 1,51 & $12,92 \mathrm{a}$ & 3,00 \\
\hline
\end{tabular}

DE: Desviación Estándar. Letras distintas en la misma columna indican diferencias significativas $(p<=0,05)$

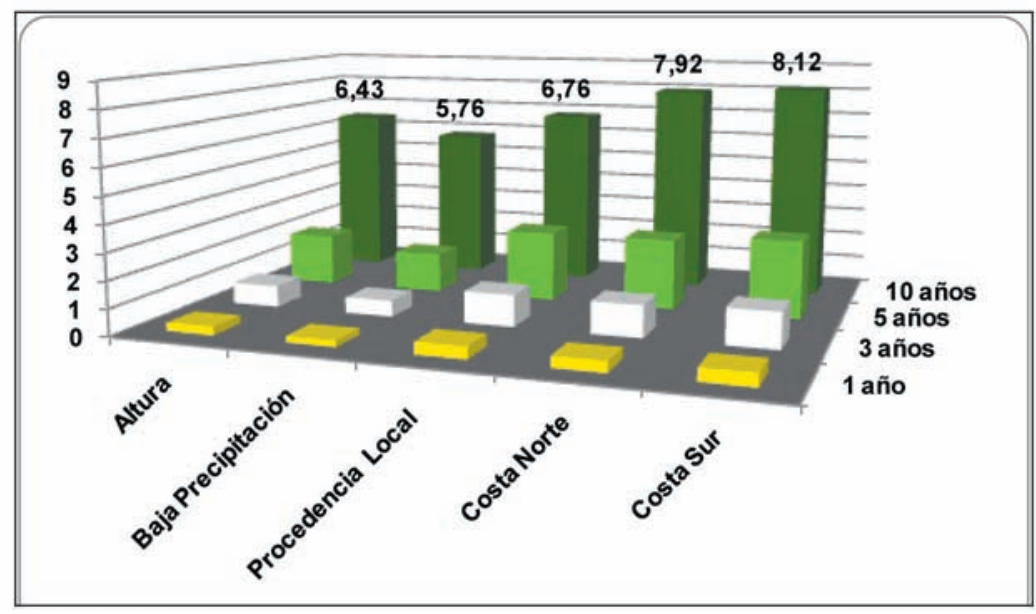

Figura $N^{\circ} 2$

\section{ALTURA (m) SEGÚN EDAD (años) POR GRUPOS DE PROCEDENCIAS}

Al analizar los incrementos medios anuales, tanto para la altura como para el DAP, se aprecia que las tendencias son las mismas que las observadas para los otros parámetros, siendo el grupo de procedencia A4 el que presenta los mayores valores, identificada como Chetco o zona de semillas 090 - 082. El grupo Baja precipitación presenta los valores más bajos, siendo estas diferencias estadísticamente significativas respecto a los otros grupos.

En cuanto al comportamiento de las procedencias, se observa en el Cuadro $\mathrm{N}^{\circ} 3$ y Figura $\mathrm{N}^{\circ} 3$ que las procedencias de la zona Costa Sur presentan los mayores valores de altura y DAP y las procedencias de la zona Baja precipitación presentan los menores valores.

Las diferencias de altura entre las procedencias en el año 2010 fueron de 3,08 m (8,42 - 5,34 m) y las de DAP de 5,44 cm $(13,43-7,99 \mathrm{~cm})$. Respecto del comportamiento de la 
procedencia local, esta presentó una altura menor en 1,66 m respecto a la procedencia A4 de la región Costa Sur, en el DAP esta diferencia fue de $2,24 \mathrm{~cm}$ en relación a la misma procedencia A4, con el mayor valor.

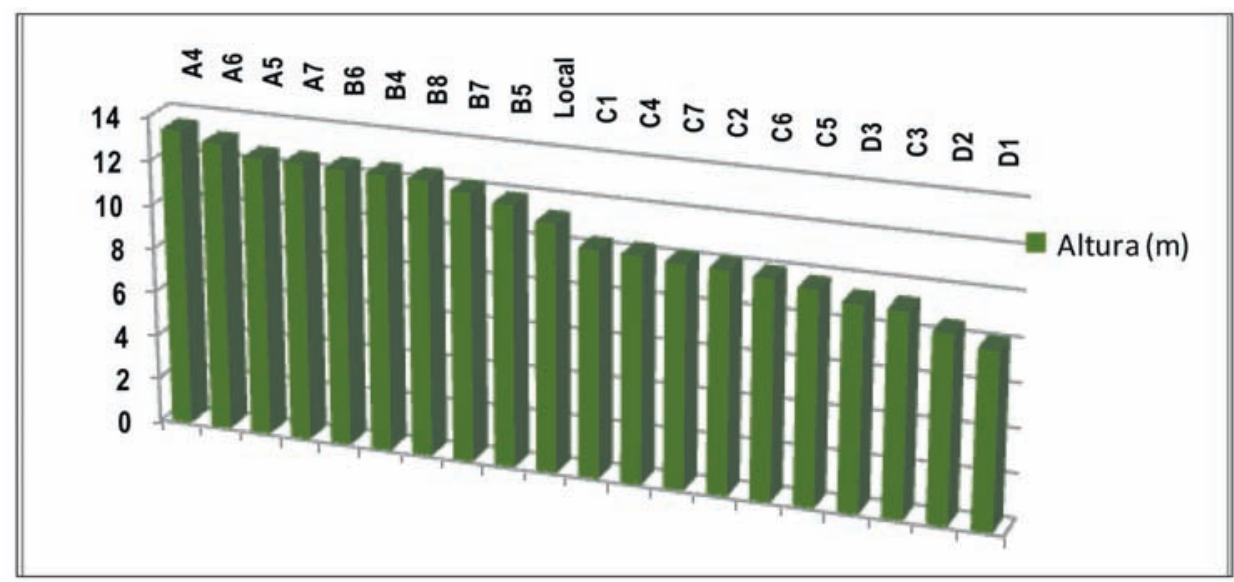

Figura $\mathrm{N}^{\circ} 3$

ALTURA SEGÚN PROCEDENCIAS 10 AÑOS EDAD 
Cuadro $\mathrm{N}^{\circ} 3$

COMPARACIONES MÚLTIPLES PARA LAS VARIABLES ALTURA Y DAP POR PROCEDENCIA

\begin{tabular}{|c|c|c|c|c|c|c|c|c|c|c|}
\hline Procedencia & $\begin{array}{c}\text { Altura } \\
\mathbf{2 0 0 1} \\
(\mathbf{m})\end{array}$ & DE & $\begin{array}{c}\text { Altura } \\
\mathbf{2 0 0 3}\end{array}$ & $\mathbf{m})$ & $\begin{array}{c}\text { DE } \\
\mathbf{2 0 0 5} \\
\mathbf{( m )}\end{array}$ & DE & $\begin{array}{c}\text { Altura } \\
\mathbf{2 0 1 0}\end{array}$ & DE & $\begin{array}{c}\text { DAP } \\
\mathbf{2 0 1 0} \\
\mathbf{( c m})\end{array}$ & DE \\
\hline A4 & 0,49 & 0,15 & 1,35 & 0,45 & 3,01 & 0,78 & $8,42 \mathrm{a}$ & 1,61 & $13,43 \mathrm{a}$ & 3,32 \\
\hline A5 & 0,47 & 0,13 & 1,28 & 0,41 & 2,79 & 0,71 & $7,94 \mathrm{c}$ & 1,41 & $12,58 \mathrm{c}$ & 3,04 \\
\hline A6 & 0,46 & 0,13 & 1,37 & 0,42 & 2,95 & 0,70 & $8,25 \mathrm{~b}$ & 1,54 & $13,05 \mathrm{~b}$ & 2,76 \\
\hline A7 & 0,46 & 0,12 & 1,36 & 0,37 & 2,87 & 0,60 & $7,84 \mathrm{c}$ & 1,38 & $12,57 \mathrm{c}$ & 2,69 \\
\hline B4 & 0,43 & 0,12 & 1,21 & 0,39 & 2,73 & 0,68 & $7,81 \mathrm{c}$ & 1,46 & $12,48 \mathrm{c}$ & 2,93 \\
\hline B5 & 0,42 & 0,12 & 1,17 & 0,40 & 2,56 & 0,66 & $7,57 \mathrm{~d}$ & 1,75 & $11,79 \mathrm{~d}$ & 3,18 \\
\hline B6 & 0,40 & 0,13 & 1,20 & 0,43 & 2,62 & 0,72 & $8,19 \mathrm{~b}$ & 1,67 & $12,49 \mathrm{c}$ & 2,91 \\
\hline B7 & 0,39 & 0,12 & 1,17 & 0,39 & 2,63 & 0,66 & $7,84 \mathrm{c}$ & 1,72 & $12,12 \mathrm{~d}$ & 2,90 \\
\hline B8 & 0,37 & 0,12 & 1,17 & 0,39 & 2,68 & 0,65 & $8,04 \mathrm{c}$ & 1,44 & $12,45 \mathrm{c}$ & 2,56 \\
\hline C1 & 0,33 & 0,12 & 0,85 & 0,38 & 2,02 & 0,66 & $6,33 \mathrm{~g}$ & 1,59 & $10,24 \mathrm{e}$ & 3,24 \\
\hline C2 & 0,33 & 0,10 & 0,83 & 0,35 & 1,92 & 0,61 & $6,53 \mathrm{f}$ & 1,51 & $10,04 \mathrm{e}$ & 3,31 \\
\hline C3 & 0,30 & 0,09 & 0,77 & 0,34 & 1,81 & 0,62 & $6,00 \mathrm{~h}$ & 1,67 & $9,21 \mathrm{~g}$ & 3,31 \\
\hline C4 & 0,28 & 0,09 & 0,74 & 0,34 & 1,81 & 0,62 & $6,78 \mathrm{e}$ & 2,27 & $10,21 \mathrm{e}$ & 4,43 \\
\hline C5 & 0,32 & 0,11 & 0,80 & 0,40 & 1,88 & 0,75 & $6,21 \mathrm{~g}$ & 1,87 & $9,65 \mathrm{f}$ & 3,69 \\
\hline C6 & 0,29 & 0,11 & 0,82 & 0,36 & 1,98 & 0,66 & $6,48 \mathrm{f}$ & 1,57 & $9,88 \mathrm{e}$ & 3,26 \\
\hline C7 & 0,29 & 0,10 & 0,82 & 0,36 & 1,96 & 0,63 & $6,57 \mathrm{f}$ & 1,65 & $10,06 \mathrm{e}$ & 3,21 \\
\hline D1 & 0,22 & 0,07 & 0,54 & 0,25 & 1,34 & 0,53 & $5,34 \mathrm{k}$ & 1,79 & $7,99 \mathrm{i}$ & 3,47 \\
\hline D2 & 0,22 & 0,09 & 0,60 & 0,32 & 1,46 & 0,65 & $5,61 \mathrm{i}$ & 1,87 & $8,48 \mathrm{~h}$ & 3,88 \\
\hline D3 & 0,24 & 0,09 & 0,65 & 0,30 & 1,61 & 0,61 & $6,22 \mathrm{~g}$ & 1,69 & $9,25 \mathrm{~g}$ & 3,45 \\
\hline Proc. Local & $\mathbf{0 , 4 5}$ & $\mathbf{0 , 1 5}$ & $\mathbf{1 , 1 9}$ & $\mathbf{0 , 4 9}$ & $\mathbf{2 , 6 3}$ & $\mathbf{0 , 8 4}$ & $\mathbf{6 , 7 6} \mathrm{e}$ & $\mathbf{1 , 6}$ & $\mathbf{1 1 , 1 9} \mathrm{d}$ & $\mathbf{5 , 5 8}$ \\
\hline
\end{tabular}

DE: Desviación Estándar; Letras distintas en la misma columna indican diferencias significativas $(p<=0,05)$ 


\section{REFERENCIAS}

Aguirre, J. y Wrann, J., 1989: Selección de procedencias de pino oregon (Pseudotsuga menziesii (Mirbel) Franco) en la zona sur de Chile. Ciencia e investigación Forestal 3(6): 7384.

Cown, D. J. 1999: New Zealand Radiata Pine and Douglas-fir. Suitability for Processing. FRI Bulletin 168.. Forest Research Institute, Rotorua, New Zealand 74 p.

Droppelmann, J. 1986: Evaluación de un ensayo de procedencia de pino Oregon (Pseudotsuga menziesii (Mirbel) Franco) de 17 años de edad. Tesis de Grado. Facultad de Ciencias Forestales, Universidas Austral de Chile. Valdivia, Chile.

Gutiérrez, B. (ed.), 2006. Propagación, respaldo y evaluación de desempeño de material genético selecto de pino oregón: Síntesis de los resultados del proyecto INFOR-INNOVA Chile "Propagación de genotipos de interés comercial de pino oregón". 152 p.

INFOR, 2010. Anuario Forestal 2010. Boletín Estadístico N²128. Instituto Forestal, Chile.

Miller, J. and Knowles, F., 1994. Douglas-fir, Pseudotsuga menziesii (Mirbel) Franco. Introduced Forest Trees in New Zealand: Recognition, Role, and Seed Sourse.FRI Bulletin 124. 38p.

Quiroz, I. y Rojas, Y., 2003. Pino ponderosa y pino oregón. Coníferas para el sur de Chile. Instituto Forestal, Valdivia. 302 p.

Santibáñez, F. y Uribe, J., 1993. Atlas Agroclimático de Chile: Regiones VIII y IX. Universidad de Chile, Santiago, Chile. Facultad de Ciencias Agrarias y Forestales, Laboratorio de Agroclimatología. 66 p.

Tosso, J., 1985. Suelos Volcánicos de Chile. Ministerio de Agricultura, Instituto de Investigaciones Agropecuarias. Santiago, Chile. 723 p.

Toval, G., Vega G., Puerto, G. and Jenkinson. A., 1993: Screening Douglas-fir for rapid early growth in Common tests in Spain. USDA Forest Service.Pacific Southwest Research Station. General Technical Report PSW-GRT-146-.43p.

Vega, G., Rodríguez, R., Arenas, S., García, S., Mansilla, P., Vega, P. y Ruiz, A., 1998. Manual de Selvicultura de Pino oregón. Disponible en: http://www.agrobyte.com/agrobyte/ publicaciones/oregón/indice.html (Acceso el 1-julio-2010). 
